Journal of Agricultural Sciences
(Tarim Bilimleri Dergisi)

\title{
Determination of Morphological, Pomological and Molecular Variations among Apples in Niğde, Turkey using iPBS Primers
}

\author{
Orkun GENCER ${ }^{\text {a* }}$ D, Sedat SERÇEa ${ }^{\mathrm{ID}}$

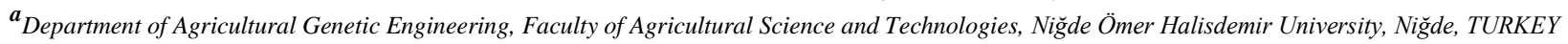

ARTICLE INFO

Research Article

Corresponding Author: Orkun GENCER, E-mail: orkungencer@ohu.edu.tr

Received: 08 February 2021 / Revised: 21 May 2021 / Accepted: 22 May 2021 / Online: 25 March 2022

\section{ABSTRACT}

In addition to morphological and pomological techniques, the molecular analysis produces more information for diversity studies. Recently, the iPBS marker system is one of the techniques and a new marker system for apple studies. In this study, morphological, pomological, and molecular characteristics of local apples were investigated in 48 different samples from 29 different rural areas with varying altitudes between $1125-1726 \mathrm{~m}$ in Niğde, Turkey. Fruit size, fruit weight, the color of fruit peel, total soluble solids content, fruit flesh firmness characteristics are important in terms of yield, quality, storage, transportation and attractiveness. According to the pomological results from these traits,
CKR2, DMR3, CLL, HCB2, YSL, ULG, ELM1, ICM have been found to superior among genotypes. In order to molecular results, the similarity of the samples varies between 0.61-1.00, under the light of this result, molecular data differentiated all individuals used in the study except one pair. Molecular data displayed that these differences were caused by genotypic differences as well as environmental conditions. This study has contributed further information about the usage of iPBS primers on apple. To protect the plant material used in the study, a collection orchard was established with genotypes. To conclude, the findings are expected to shape future breeding studies.

Keywords: Malus domestica, Genetic resource, Genetic diversity, Molecular analysis, Polymorphism

(C) Ankara University, Faculty of Agriculture

\section{Introduction}

Apple (Malus domestica Borhk.), which is cultivated all over the world, has great diversity and the number of known cultivars are more than 6500 and, particularly 600 cultivars in Turkey (Hancock 2012). Central Asia, Caucasus, and Turkey were the center of origin for apples. Kayseri and Ağrı provinces are two major diversity points in Turkey (Ozbek 1978). According to FAO 2020 stats, Turkey has an important position in world apple production, ranked fourth amongst China, United States, and Poland. According to TUIK 2020 stats, Niğde is one of the three major provinces in Turkey's apple production and $81.0 \%$ of Niğde's apple production is covered by Central and Bor districts. Niğde has important advantages for apple production as follows: the flat land structure of the province is suitable for establishing orchards, land prices are cheaper than other provinces producing apples in Turkey, fewer diseases have been seen such as black spots by low humidity and better-colored fruits by the temperature difference between day/night (Anonymous 2014).

The key to high yields is application of modern agricultural techniques (Demir \& Doğan 2020). Although new and modern apple orchards have started to establish in Niğde recently, a lot of established orchards that are using old agricultural practices for many years. In this case, the size and color of fruits are non-uniform. The local non-registered cultivars that are widely cultivated in the province are 'Amasya', 'Orak Apple', 'Demir Apple', 'Tavşanbaşı', 'Arapkızı' and 'Hüryemez'. Also, in recent years, popular cultivars such as 'Granny Smith', 'Fuji', 'Red Chief', 'Mondial Gala', 'Super Chief', 'Scarlet Spur' have been cultivated in the newly established orchards by a dwarf and semi-dwarf rootstocks.

Morphological and pomological characteristics are environmentally affected due to the nature of it. This information is not enough to effectively differentiate individuals. On the other hand, genetic characteristics supply more stable information for this purpose. After the development of molecular techniques and utilization of plant breeding, the researcher got a chance to clarify their phenotypic data with genetic data. Thus, researchers enhance the accuracy of their studies.

The selection of parents is always an important process for plant breeders and genetic similarity data helps to make more precise decisions about this step. Numerous DNA marker systems are available to show genetic variations among plants. Simple sequence repeat (SSR) (Hokanson et al. 1998; Hokanson et al. 2001; Zhang et al. 2007, Bakır et al. 2019), inter-simple sequence repeat (ISSR) (Smolik \& Krzysztoszek 2010), RAPD (random amplified polymorphic DNA) (Dunemann et al. 1994; Zhou \& Li 
2000) and amplified fragment length polymorphism (AFLP) (Kenis \& Keulemans 2005) are just some of them. AFLP and SSR systems are considered as a substantial way to indicate genetic variations in plants but these methods demand more costly instruments, usage of advanced steps in the process, and a significant amount of time compared to others.

Inter-primer binding sites (iPBS) retrotransposon marker system was developed by Kalendar et al. (2010) for plants and as well as animal kingdoms. The importance of this marker system is not to require sequence knowledge about plant/animal of interest. Retrotransposons can relocate themselves to the genome via copying. This mechanism usually ended up with different outcomes but the main result, expansion of genome size as well as genetic variation. For this reason, retrotransposons are accepted as valuable tools among other molecular marker systems.

Other studies on fruits have been carried out using iPBS. Guo et al. (2013), conducted a study with 35 grape varieties to evaluate for their molecular diversity associated with iPBS markers. In their experiment, 99 polymorphic DNA bands were produced with 15 iPBS primers. They indicated iPBS markers suitable for genetic diversity studies on grapes. Rovna et al. (2020), carried out a study with Rosa canina fruits to determine their genome size, iPBS profiles as well as antioxidant and antimicrobial actives. Their results suggested that iPBS markers provide favorable techniques for evaluating the genetic variability of Rosa canina.

Kuras et al. (2013), performed an experiment with 5 different DNA marker techniques to distinguish five different apple cultivars and their spots. According to their results, iPBS primers produced many polymorphic DNA output that has been able to distinguish five progenitor cultivars however not many polymorphic bands were sport specific. Correct utilization of iPBS marker system has been shown the power to identify different apple cultivars.

In general, the iPBS marker system is economic compared to other marker systems (which is an important feature especially for some countries), including screening huge part of plant genomes, usage for a different living organism, and user friendly to researchers (Kuras et al. 2013; Demirel et al. 2018; Milovanov et al. 2019).

Apple has already a growing market in Turkey. The current situation can be improved by eliminating known issues and evaluating the potential of local cultivars. This is the first study conducted in Niğde with these local apple genotypes since the study of Eltez \& Kaska (1985). This study aims to evaluate the situation in the manner of genetic diversity to apple on the region of interest. For archive to this goal, iPBS markers utilized as a main tool and results also shown a convenient method to apple. Some studies were conducted on apple cultivars \& mutants with iPBS markers, but this is the first study to the utilization of iPBS marker system on apple genotypes. This study is also the first step of the future breeding program in the region.

\section{Material and Methods}

The study was carried out with 48 different apple trees in 29 rural areas of Niğde, Turkey in 2018-2019 (Table 1). Altitude values of trees vary between 1125-1726 m (Table 1). 'Super Chief', 'Fuji', 'Granny Smith' cultivars taken from Niğde Ömer Halisdemir University Faculty of Agricultural Sciences and Technologies Research and Application Orchard was used as a control group for pomological analysis. In addition to pomological control groups 'Golden Delicious', 'Scarlet Spur' cultivars taken from the application orchard and known local apples called 'green sour apple', 'sour summer apple', 'red sour apple', 'golden seed', 'rabbit head', 'bowl apple', 'red summer apple' also used in the control group of molecular analysis. 
Table 1- Information about plant materials (apple trees) and locations

\begin{tabular}{|c|c|c|c|}
\hline Tree codes & Name of locations & GPS data & Elevation (meter) \\
\hline KMR & Kemerhisar & $37^{\circ} 49^{\prime} 56.9^{\prime \prime} \mathrm{N} 34^{\circ} 35^{\prime} 29.3^{\prime \prime E}$ & 1125 \\
\hline $\mathrm{BHC}$ & Bahçeli & $37^{\circ} 50^{\prime} 06.7^{\prime \prime N} 34^{\circ} 36^{\prime} 39.5^{\prime \prime E}$ & 1147 \\
\hline SZL & Sazlica & $37^{\circ} 54^{\prime} 04.3^{\prime \prime} \mathrm{N} 34^{\circ} 38^{\prime} 34.8^{\prime \prime} \mathrm{E}$ & 1211 \\
\hline HLC & Halaç & $37^{\circ} 49^{\prime} 39.0^{\prime \prime} \mathrm{N} 34^{\circ} 41^{\prime} 19.3^{\prime \prime E}$ & 1297 \\
\hline $\mathrm{KRC}$ & Karacaören & $37^{\circ} 48^{\prime} 04.1^{\prime \prime N} 34^{\circ} 43^{\prime} 36.9^{\prime \prime} \mathrm{E}$ & 1487 \\
\hline KLV & Kilavuz & $37^{\circ} 47^{\prime} 53.8^{\prime \prime} \mathrm{N} 34^{\circ} 46^{\prime} 06.7^{\prime \prime} \mathrm{E}$ & 1571 \\
\hline HVZ & Havuzlu & $37^{\circ} 46^{\prime} 38.0^{\prime \prime} \mathrm{N} 34^{\circ} 37^{\prime} 59.1^{\prime \prime E}$ & 1213 \\
\hline PST & Postall1 & $37^{\circ} 43^{\prime} 46.9^{\prime \prime} \mathrm{N} 34^{\circ} 45^{\prime} 17.0^{\prime \prime} \mathrm{E}$ & 1394 \\
\hline DGR & Değirmenli & $38^{\circ} 02^{\prime} 54.4^{\prime \prime} \mathrm{N} 34^{\circ} 54^{\prime} 06.4^{\prime \prime} \mathrm{E}$ & 1494 \\
\hline DND & Dündarlı & $38^{\circ} 05^{\prime} 28.7^{\prime \prime} \mathrm{N} 35^{\circ} 09^{\prime} 54.4^{\prime \prime} \mathrm{E}$ & 1326 \\
\hline CKR1 & Çukurbağ & $37^{\circ} 50^{\prime} 09.6^{\prime \prime} \mathrm{N} 35^{\circ} 03^{\prime} 25.8^{\prime \prime} \mathrm{E}$ & 1484 \\
\hline CKR2 & Çukurbağ & $37^{\circ} 50^{\prime} 08.7^{\prime \prime} \mathrm{N} 35^{\circ} 03^{\prime} 33.2^{\prime \prime} \mathrm{E}$ & 1493 \\
\hline CKR3 & Çukurbağ & $37^{\circ} 49^{\prime} 60.0^{\prime \prime} \mathrm{N} 35^{\circ} 03^{\prime} 27.7^{\prime \prime} \mathrm{E}$ & 1499 \\
\hline CKR4 & Çukurbağ & $37^{\circ} 50^{\prime} 07.1^{\prime \prime} \mathrm{N} 35^{\circ} 03^{\prime} 21.4^{\prime \prime} \mathrm{E}$ & 1480 \\
\hline CKR5 & Çukurbağ & $37^{\circ} 50^{\prime} 07.2^{\prime \prime} \mathrm{N} 35^{\circ} 03^{\prime} 10.9^{\prime \prime} \mathrm{E}$ & 1455 \\
\hline BDM1 & Bademdere & $37^{\circ} 55^{\prime} 04.7^{\prime \prime} \mathrm{N} 35^{\circ} 04^{\prime} 14.8^{\prime \prime} \mathrm{E}$ & 1601 \\
\hline BDM2 & Bademdere & $37^{\circ} 55^{\prime} 01.5^{\prime \prime} \mathrm{N} 35^{\circ} 04^{\prime} 18.1^{\prime \prime} \mathrm{E}$ & 1595 \\
\hline BDM3 & Bademdere & $37^{\circ} 54^{\prime} 58.9^{\prime \prime} \mathrm{N} 35^{\circ} 04^{\prime} 24.5^{\prime \prime} \mathrm{E}$ & 1586 \\
\hline BDM4 & Bademdere & $37^{\circ} 54^{\prime} 53.9^{\prime \prime} \mathrm{N} 35^{\circ} 04^{\prime} 24.2^{\prime \prime} \mathrm{E}$ & 1582 \\
\hline BDM5 & Bademdere & $37^{\circ} 54^{\prime} 47.8^{\prime \prime} \mathrm{N} 35^{\circ} 04^{\prime} 26.2^{\prime \prime} \mathrm{E}$ & 1576 \\
\hline PNR1 & Pınarbaş1 & $37^{\circ} 53^{\prime} 43.7^{\prime \prime} \mathrm{N} 35^{\circ} 05^{\prime} 00.8^{\prime \prime} \mathrm{E}$ & 1574 \\
\hline PNR2 & Pınarbaşı & $37^{\circ} 53^{\prime} 36.7^{\prime \prime} \mathrm{N} 35^{\circ} 05^{\prime} 15.9^{\prime \prime} \mathrm{E}$ & 1569 \\
\hline PNR3 & Pınarbaşı & $37^{\circ} 53^{\prime} 26.4^{\prime \prime} \mathrm{N} 35^{\circ} 05^{\prime} 35.5^{\prime \prime} \mathrm{E}$ & 1572 \\
\hline PNR4 & Pınarbaşı & $37^{\circ} 53^{\prime} 15.0^{\prime \prime} \mathrm{N} 35^{\circ} 06^{\prime} 02.0^{\prime \prime} \mathrm{E}$ & 1562 \\
\hline DMR1 & Demirkazık & $37^{\circ} 51^{\prime} 41.0^{\prime \prime} \mathrm{N} 35^{\circ} 05^{\prime} 31.5^{\prime \prime} \mathrm{E}$ & 1577 \\
\hline PNR5 & Pınarbaşı & 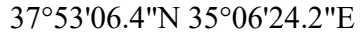 & 1598 \\
\hline DMR2 & Demirkazık & $37^{\circ} 51^{\prime} 32.2^{\prime \prime} \mathrm{N} 35^{\circ} 05^{\prime} 16.6^{\prime \prime} \mathrm{E}$ & 1558 \\
\hline DMR3 & Demirkazık & $37^{\circ} 51^{\prime} 28.7^{\prime \prime} \mathrm{N} 35^{\circ} 05^{\prime} 04.8^{\prime \prime} \mathrm{E}$ & 1545 \\
\hline DMR4 & Demirkazık & $37^{\circ} 51^{\prime} 28.4^{\prime \prime} \mathrm{N} 35^{\circ} 04^{\prime} 50.9^{\prime \prime} \mathrm{E}$ & 1556 \\
\hline DMR5 & Demirkazık & $37^{\circ} 51^{\prime} 25.4^{\prime \prime} \mathrm{N} 35^{\circ} 04^{\prime} 43.4^{\prime \prime} \mathrm{E}$ & 1560 \\
\hline CLL & Celaller & $37^{\circ} 48^{\prime} 34.6^{\prime \prime} \mathrm{N} 34^{\circ} 56^{\prime} 09.5^{\prime \prime} \mathrm{E}$ & 1687 \\
\hline $\mathrm{BRC}$ & Burç & $37^{\circ} 48^{\prime} 12.9^{\prime \prime} \mathrm{N} 34^{\circ} 59^{\prime} 11.4^{\prime \prime} \mathrm{E}$ & 1445 \\
\hline ELG & Elekgölü & 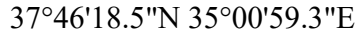 & 1365 \\
\hline KVL1 & Kavlaktepe & $37^{\circ} 59^{\prime} 29.8^{\prime \prime} \mathrm{N} 35^{\circ} 05^{\prime} 34.0^{\prime \prime} \mathrm{E}$ & 1671 \\
\hline KVL2 & Kavlaktepe & $37^{\circ} 59^{\prime} 00.8^{\prime \prime} \mathrm{N} 35^{\circ} 05^{\prime} 34.9^{\prime \prime} \mathrm{E}$ & 1726 \\
\hline HCB1 & Hacıbeyli & $38^{\circ} 07^{\prime} 17.7^{\prime \prime} \mathrm{N} 35^{\circ} 09^{\prime} 19.9^{\prime \prime} \mathrm{E}$ & 1280 \\
\hline $\mathrm{HCB} 2$ & Hacıbeyli & $38^{\circ} 07^{\prime} 05.3^{\prime \prime} \mathrm{N} 35^{\circ} 09^{\prime} 28.9^{\prime \prime} \mathrm{E}$ & 1283 \\
\hline DKL & Dikilitaş & $38^{\circ} 06^{\prime} 56.9^{\prime \prime} \mathrm{N} 35^{\circ} 04^{\prime} 25.3^{\prime \prime} \mathrm{E}$ & 1435 \\
\hline YSL & Yeşilova & $38^{\circ} 03^{\prime} 31.3^{\prime \prime} \mathrm{N} 34^{\circ} 49^{\prime} 58.3^{\prime \prime} \mathrm{E}$ & 1388 \\
\hline ULG & Uluağaç & $38^{\circ} 02^{\prime} 34.6^{\prime \prime} \mathrm{N} 34^{\circ} 50^{\prime} 20.2^{\prime \prime} \mathrm{E}$ & 1435 \\
\hline GMS & Gümüşler & $37^{\circ} 59^{\prime} 56.2^{\prime \prime} \mathrm{N} \mathrm{34} 4^{\circ} 45^{\prime} 59.7^{\prime \prime} \mathrm{E}$ & 1344 \\
\hline HMM & Himmetli & $38^{\circ} 02^{\prime} 08.8^{\prime \prime} \mathrm{N} 34^{\circ} 56^{\prime} 32.7^{\prime \prime E}$ & 1552 \\
\hline ELM1 & Elmalı & $38^{\circ} 01^{\prime} 52.1^{\prime \prime} \mathrm{N} 34^{\circ} 57^{\prime} 41.6^{\prime \prime} \mathrm{E}$ & 1603 \\
\hline ELM2 & Elmalı & $38^{\circ} 01^{\prime} 12.8^{\prime \prime} \mathrm{N} 34^{\circ} 58^{\prime} 29.0^{\prime \prime} \mathrm{E}$ & 1605 \\
\hline $\mathrm{KCP}$ & Kocapinar & $38^{\circ} 01^{\prime} 37.2^{\prime \prime} \mathrm{N} 35^{\circ} 05^{\prime} 43.2^{\prime \prime} \mathrm{E}$ & 1571 \\
\hline EYN & Eynelli & $37^{\circ} 53^{\prime} 51.3^{\prime \prime} \mathrm{N} 35^{\circ} 03^{\prime} 46.9^{\prime \prime} \mathrm{E}$ & 1531 \\
\hline $\mathrm{ICM}$ & İçmeli & $38^{\circ} 03^{\prime} 24.2^{\prime \prime} \mathrm{N} 35^{\circ} 05^{\prime} 49.6^{\prime \prime} \mathrm{E}$ & 1519 \\
\hline YLT & Yelatan & 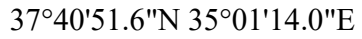 & 1320 \\
\hline
\end{tabular}

\subsection{Morphological analysis}

Morphological analysis was conducted before harvest (Tijskens et al. 2007). Tree habit (Upright, spreading, drooping, weeping), tree trunk diameter (Measured from $15 \mathrm{~cm}$ above to ground) and height of tree trunk (Measured from grafting point to first branches) $(\mathrm{cm})$, one-year-old shoot length $(\mathrm{cm})$, leaf blade attitude in relation to shoot (Upwards, outwards, downwards), leaf blade length and width ( $\mathrm{mm}$ ), leaf blade incisions of margin (Crenate, bicrenate, serrate type 1, serrate type 2, biserrate), petiole length $(\mathrm{mm})$ and fruit general shape (Cylindrical waisted, conic, ovoid, cylindrical, ellipsoid, globose, obolid) measurements was collected for morphological analysis (UPOV 2005). All measurements except tree habit, tree trunk diameter and height of tree trunk, held with 3 repeats, each repeat subjected to 5 related plant materials in total 15 . 


\subsection{Pomological analysis}

The pomological analysis was carried out for 3 repeats, each repeat contains 5 fruit in total 15 ripe fruits for each tree. Fruit height and diameter $(\mathrm{mm})$, fruit weight $(\mathrm{g})$, depth of stalk cavity $(\mathrm{mm})$, fruit skin color (Measured by KONICA MINOLTA CM700d Spectrophotometer), the color of flesh (White, cream, yellowish, greenish, pinkish, reddish), number of seeds, the aperture of locules (Closed or slightly open, moderately open, fully open), firmness of flesh (Measured by handheld fruit penetrometer) $\left(\mathrm{kg} / \mathrm{cm}^{2}\right), \mathrm{pH}$ (Measured by VWR pHenomenal 1000L digital ph meter) and total soluble solids content (Measured by KRÜSS AR2008 digital refractometer) measurements were collected for pomological analysis (UPOV 2005).

\subsection{Molecular analysis with iPBS primers}

DNA extraction was conducted by the CTAB method from 3-5 young leaves taken from each tree (Dellaporta et al. 1983). The concentrations of the DNAs were then determined by the Quawell Q5000 UV-Vis Spectrophotometer and diluted to $5 \mathrm{ng} / \mathrm{uL}$. IPBS primers were used in molecular marker analyzes (Table 4).

In total 60 apple genotypes (12 of them belong to the control group) were evaluated with 15 iPBS markers developed by Kalendar et al. (2010) to show the genetic diversity of these apple genotypes (Table 4). Diluted DNAs was amplified by PCR. The PCR was performed in a $25 \mu \mathrm{L}$ reaction mixture containing $5 \mu \mathrm{L}$ DNA $(5 \mathrm{ng} / \mu \mathrm{L}), 2.5 \mu \mathrm{L} 10 \mathrm{X}$ DreamTaq PCR buffer, 0.375 $\mu \mathrm{L}$ dNTPs, $3 \mu \mathrm{L}$ primer for 18 bp primers \& $5 \mu \mathrm{L}$ primer for $12-13$ bp primers, $0.2 \mu \mathrm{L}$ DreamTaq DNA polymerase.

Following initial denaturation at $95^{\circ} \mathrm{C}$ for $3 \mathrm{~min}, \mathrm{PCR}$ was conducted in order of amplification for 35 cycles with denaturation at $95{ }^{\circ} \mathrm{C}$ for $15 \mathrm{~s}$ annealing at $50-63$ (specific to primers in Table 4) for $60 \mathrm{~s}$ and extension at $72{ }^{\circ} \mathrm{C}$ for 2 min. Lastly, the final extension was completed in a stage of $72{ }^{\circ} \mathrm{C}$ for $7 \mathrm{~min}$. Products of PCR electrophoresed at 60 volts for 2.5 hours on a $1.8 \%$ agarose gel prepared with $1 \mathrm{X}$ TAE, stained with ethidium bromide for 30 minutes, and then viewed with Bio-Rad Gel Doc ${ }^{\mathrm{TM}}$ $\mathrm{XR}+$ gel imaging system. PCR or electrophoresis process repeated if it requires to get a clearer image of gel that suitable for scoring.

\subsection{Statistical analysis}

The SAS program was used for statistical analysis of pomological data (SAS 2005). The Duncan's Multiple Range Test was used to differentiate the mean values of the significant values $(\mathrm{P}<0.05)$. In the evaluation of the data obtained as a result of molecular analysis, the result file was created in a binary number system according to whether the molecular markers used in the gel images were shown as (1) or not (0). From these results, a similarity matrix was created with the appropriate Jaccard method and then data clustering and TKoA analyzes were applied by using the NTSYS program (Rohlf 1998). As a result of the clustering analysis, the dendrogram was generated by the UPGMA method. Mantel's matrix correspondence test was used to test the agreement of the dendrogram with the similarity matrix. Polymorphism information content (PIC) was calculated according to the formula given by Hinze et al. (2015) due to the iPBS makers system is a dominant marker. Principal Coordinates Analysis (PCoA) was performed using PAST 4.03 software (Hammer et al. 2001).

\section{Results}

\subsection{Morphological results}

Duncan's Multiple Range Test was applied to quantitative data of morphological observations. According to the morphologic results, no 'upright' types were found in any tree habit characteristics, and 'drooping' is the most common type 20 times. The highest values (significant in statically) found in tree trunk diameter was KVL2 (46.63), the height of tree trunk was BDM1 $(148.5) \mathrm{cm}$, one-year-old shoot length was BDM4 $(114.22 \mathrm{~cm})$. 'Downwards' type was not found in leaf blade attitude in relation to shoot characteristics and the 'upwards' type was the most common feature with 33 times. The highest values (statically significant) found in leaf blade length was ULG $(87.57 \mathrm{~cm})$, leaf blade width was KCP $(47.59 \mathrm{~cm})$. Among leaf blade incisions of margin characteristics 'crenate' and 'bicrenate' type was not found, 'serrate type 2' is the most common type with 25 times. The highest values (statically significant) found in petiole length were KVL1 $(36.65 \mathrm{~cm})$. Results of fruit general shape indicated that only 'globose' and 'obloid' features were founded among the genotypes and 'obloid' was the most common type with 33 times (Table 2).

The results obtained from the morphological analyzes revealed the expected characteristics and values of the local Niğde, Turkey apples, and provided a basis for further characterization studies. 
Table 2- Morphological analysis results

\begin{tabular}{|c|c|c|c|c|c|c|c|c|c|c|}
\hline $\begin{array}{l}\text { Tree } \\
\text { codes }\end{array}$ & $\begin{array}{l}\text { Tree } \\
\text { habit }\end{array}$ & $\begin{array}{l}\text { Tree trunk } \\
\text { diameter }\end{array}$ & $\begin{array}{l}\text { Height of } \\
\text { tree trunk }\end{array}$ & $\begin{array}{l}\text { One year old } \\
\text { shoot length }\end{array}$ & $\begin{array}{l}\text { Leaf blade attitude } \\
\text { in relation to shoot }\end{array}$ & $\begin{array}{l}\text { Leaf blade } \\
\text { length }\end{array}$ & $\begin{array}{l}\text { Leaf blade } \\
\text { width }\end{array}$ & $\begin{array}{l}\text { Leaf blade } \\
\text { incisions of margin }\end{array}$ & $\begin{array}{l}\text { Petiole } \\
\text { length }\end{array}$ & $\begin{array}{l}\text { Fruit general } \\
\text { shape }\end{array}$ \\
\hline KMR & Spreading & 32.79 & 83 & 51.56 & Upwards & 71.8 & 45.08 & Serrate type 2 & 30.91 & Globose \\
\hline $\mathrm{BHC}$ & Drooping & 40.33 & 106.5 & 60.32 & Upwards & 68.25 & 39.58 & Serrate type 2 & 33.51 & Obolid \\
\hline SZL & Spreading & 13.05 & 92.5 & 30 & Upwards & 58.27 & 32.44 & Serrate type 2 & 24.79 & Obolid \\
\hline HLC & Drooping & 15.37 & 100.3 & 70.28 & Upwards & 63.52 & 40.1 & Biserrate & 27.25 & Obolid \\
\hline $\mathrm{KRC}$ & Weeping & 32.75 & 79 & 83.12 & Upwards & 62.71 & 35.79 & Biserrate & 27.27 & Obolid \\
\hline KLV & Weeping & 27.28 & 112 & 51.82 & Upwards & 70.72 & 40.93 & Serrate type 1 & 33.3 & Obolid \\
\hline HVZ & Weeping & 33.01 & 108 & 73.94 & Outwards & 66.56 & 40.87 & Biserrate & 31.6 & Obolid \\
\hline PST & Weeping & 27.95 & 84 & 53.98 & Upwards & 74.84 & 38.82 & Serrate type 2 & 26.64 & Obolid \\
\hline DGR & Drooping & 38.13 & 125.7 & 63.8 & Upwards & 73.75 & 40.67 & Serrate type 2 & 31.37 & Obolid \\
\hline DND & Drooping & 22.38 & 108.5 & 44.82 & Upwards & 72.15 & 40.02 & Serrate type 2 & 29.51 & Globose \\
\hline CKR1 & Drooping & 20.18 & 123 & 49.52 & Upwards & 64.56 & 39.09 & Biserrate & 32.31 & Obolid \\
\hline CKR2 & Drooping & 20.72 & 74.3 & 38.66 & Upwards & 70.03 & 36.28 & Serrate type 1 & 27.81 & Obolid \\
\hline CKR3 & Drooping & 39.57 & 82.2 & 107.5 & Outwards & 71.21 & 36.41 & Biserrate & 29.17 & Obolid \\
\hline CKR4 & Weeping & 23.62 & 132.5 & 43.08 & Outwards & 77.86 & 44.15 & Biserrate & 35.01 & Obolid \\
\hline CKR5 & Spreading & 26.74 & 116 & 60.46 & Outwards & 80.93 & 42.16 & Biserrate & 34.02 & Globose \\
\hline BDM1 & Spreading & 30.46 & 148.5 & 41.24 & Upwards & 66.83 & 36.59 & Serrate type 1 & 31.8 & Obolid \\
\hline BDM2 & Drooping & 33.61 & 132 & 71.36 & Upwards & 73.79 & 44.8 & Serrate type 2 & 30.17 & Obolid \\
\hline BDM3 & Drooping & 36.06 & 50.4 & 65.78 & Upwards & 78.49 & 39.8 & Biserrate & 29.93 & Globose \\
\hline BDM4 & Weeping & 20.21 & 64 & 114.22 & Outwards & 73.55 & 41.28 & Serrate type 2 & 34.17 & Obolid \\
\hline BDM5 & Weeping & 23.87 & 122 & 62.94 & Outwards & 72.47 & 40.4 & Serrate type 2 & 36.21 & Obolid \\
\hline PNR1 & Spreading & 33.9 & 123 & 85.24 & Upwards & 69.58 & 41.89 & Serrate type 2 & 29.73 & Globose \\
\hline PNR2 & Drooping & 24.67 & 112.4 & 51 & Upwards & 72.11 & 44.26 & Serrate type 2 & 35.48 & Obolid \\
\hline PNR3 & Spreading & 28.74 & 97.4 & 81.44 & Upwards & 76.93 & 46.23 & Serrate type 2 & 35.02 & Obolid \\
\hline PNR4 & Drooping & 32.79 & 83 & 51.56 & Upwards & 71.8 & 45.08 & Biserrate & 30.91 & Obolid \\
\hline PNR5 & Weeping & 40.33 & 106.5 & 60.32 & Upwards & 68.25 & 39.58 & Biserrate & 33.51 & Obolid \\
\hline DMR1 & Weeping & 13.05 & 92.5 & 30 & Upwards & 58.27 & 32.44 & Serrate type 1 & 24.79 & Obolid \\
\hline DMR2 & Weeping & 15.37 & 100.3 & 70.28 & Outwards & 63.52 & 40.1 & Biserrate & 27.25 & Obolid \\
\hline DMR3 & Weeping & 32.75 & 79 & 83.12 & Upwards & 62.71 & 35.79 & Serrate type 2 & 27.27 & Obolid \\
\hline DMR4 & Drooping & 27.28 & 112 & 51.82 & Upwards & 70.72 & 40.93 & Serrate type 2 & 33.3 & Obolid \\
\hline DMR5 & Drooping & 33.01 & 108 & 73.94 & Upwards & 66.56 & 40.87 & Serrate type 2 & 31.6 & Globose \\
\hline CLL & Drooping & 27.95 & 84 & 53.98 & Upwards & 74.84 & 38.82 & Serrate type 2 & 26.64 & Obolid \\
\hline BRC & Drooping & 38.13 & 125.7 & 63.8 & Outwards & 73.75 & 40.67 & Serrate type 2 & 31.37 & Obolid \\
\hline ELG & Spreading & 22.38 & 108.5 & 44.82 & Upwards & 72.15 & 40.02 & Serrate type 2 & 29.51 & Obolid \\
\hline KVL1 & Weeping & 36.48 & 87.3 & 60.7 & Upwards & 69.19 & 38.95 & Serrate type 1 & 36.65 & Obolid \\
\hline KVL2 & Drooping & 46.63 & 88.1 & 55.76 & Upwards & 71.65 & 45.84 & Serrate type 2 & 32.17 & Globose \\
\hline HCB1 & Weeping & 30.81 & 92.3 & 51.92 & Upwards & 79.4 & 43.73 & Serrate type 1 & 33.44 & Globose \\
\hline HCB2 & Drooping & 40.14 & 79.7 & 57.96 & Upwards & 71.13 & 43 & Serrate type 1 & 29.84 & Globose \\
\hline DKL & Spreading & 35.81 & 101.3 & 61.32 & Outwards & 68.43 & 40.78 & Biserrate & 30.12 & Globose \\
\hline YSL & Drooping & 23.65 & 62.1 & 67.14 & Outwards & 79.09 & 38.87 & Biserrate & 33.82 & Obolid \\
\hline ULG & Spreading & 31.83 & 79.2 & 57.68 & Outwards & 87.57 & 47.36 & Serrate type 2 & 33.48 & Globose \\
\hline GMS & Weeping & 33.3 & 79.4 & 41.06 & Outwards & 80.33 & 44.98 & Biserrate & 30.49 & Obolid \\
\hline HMM & Spreading & 21.74 & 60.4 & 76.16 & Upwards & 65.47 & 38.25 & Serrate type 2 & 23.81 & Globose \\
\hline ELM1 & Spreading & 31.26 & 73.6 & 45.64 & Outwards & 69.21 & 41.26 & Serrate type 2 & 23.5 & Globose \\
\hline ELM2 & Drooping & 37.97 & 88.6 & 54.36 & Outwards & 77.43 & 40.96 & Serrate type 2 & 33.58 & Globose \\
\hline $\mathrm{KCP}$ & Spreading & 33.84 & 120 & 62.08 & Upwards & 87.44 & 47.59 & Serrate type 2 & 36.2 & Globose \\
\hline EYN & Spreading & 38.67 & 98.7 & 71.08 & Outwards & 80.77 & 47.49 & Serrate type 2 & 34.69 & Obolid \\
\hline $\mathrm{ICM}$ & Drooping & 32.47 & 121.7 & 72.78 & Upwards & 82.24 & 40.48 & Biserrate & 30.66 & Obolid \\
\hline YLT & Spreading & 35.91 & 117.6 & 81.38 & Upwards & 83.9 & 46.46 & Serrate type 1 & 35.18 & Obolid \\
\hline
\end{tabular}

\subsection{Pomological analysis}

According to the Duncan's Multiple Range Test, the highest values (significant in statically) was found in fruit diameter CKR2 (72.64 mm), YSL (72.17 mm), ULG (72.07 mm), HCB2 (71.40 mm); fruit height ULG (66.40 mm); fruit weight ULG (154.44 g), YSL (147.50 g), HCB2 (145.50 g), CKR2 (144.04 g); depth of stalk cavity HCB2 (17.76 mm); the number of seeds SZL (10.33 pieces); firmness of flesh ELM1 $\left(9.10 \mathrm{~kg} / \mathrm{cm}^{2}\right)$; fruit skin color L value DMR3 (65.62), a value ICM (37.07), b value DMR3 (30.15), CLL (29.859). The most common types in flesh color were white with 31 samples and greenish with 7 samples. It was not found yellowish, pinkish, and reddish color flesh. The most observed type in the aperture of locules closed or slightly open with 28 samples and the fully open with the least common two samples. The highest value for pH was found in DMR5 
(3.88) and the lowest value was in DMR3 (3.05). The highest amount of total soluble solids content was seen in ICM (14.60\%) and the lowest value was found in HCB1 (10.30\%) (Table 3).

Table 3- Pomological analysis results

\begin{tabular}{|c|c|c|c|c|c|c|c|c|c|c|c|c|c|}
\hline Genoype \& cultivar & FHE & FDI & FWE & DSC & FSC-dL & FSC-da & FSC-db & CFE & NSE & ALO & FFE & $\mathrm{pH}$ & TSSC \\
\hline Fuji & 71.92 & 58.12 & 152.15 & 14.24 & 50.91 & 17.56 & 18.51 & 2 & 9.07 & 1 & 10.04 & 3.40 & 13.00 \\
\hline Granny Smith & 74.12 & 65.73 & 175.17 & 15.64 & 61.67 & 8.70 & 41.40 & 4 & 7.87 & 2 & 9.67 & 3.24 & 13.28 \\
\hline Super Chief & 75.05 & 66.78 & 181.38 & 16.39 & 38.39 & 27.39 & 13.31 & 2 & 5.73 & 2 & 6.08 & 3.51 & 14.70 \\
\hline KMR & 62.05 & 52.59 & 93.99 & 13.09 & 50.40 & 26.83 & 14.30 & 1 & 8.80 & 1 & 6.86 & 3.74 & 11.40 \\
\hline $\mathrm{BHC}$ & 60.86 & 46.44 & 81.71 & 13.13 & 66.91 & 16.39 & 26.02 & 1 & 9.00 & 1 & 6.68 & 3.61 & 11.00 \\
\hline SZL & 59.55 & 49.13 & 83.61 & 11.13 & 62.27 & 14.38 & 22.51 & 1 & 10.33 & 1 & 6.70 & 3.65 & 11.00 \\
\hline HLC & 66.20 & 57.76 & 112.19 & 13.71 & 56.16 & 20.24 & 19.18 & 1 & 7.00 & 1 & 6.99 & 3.51 & 12.60 \\
\hline KRC & 63.05 & 56.03 & 100.50 & 13.21 & 53.64 & 21.37 & 17.63 & 1 & 8.60 & 1 & 7.13 & 3.50 & 13.30 \\
\hline KLV & 61.46 & 52.43 & 88.67 & 11.81 & 59.66 & 19.70 & 21.95 & 1 & 8.60 & 1 & 7.15 & 3.71 & 13.70 \\
\hline HVZ & 59.60 & 47.78 & 79.16 & 12.35 & 56.20 & 24.00 & 18.19 & 1 & 8.47 & 1 & 7.64 & 3.50 & 13.80 \\
\hline PST & 57.52 & 49.49 & 78.10 & 11.43 & 61.34 & 12.78 & 22.89 & 1 & 8.33 & 1 & 6.82 & 3.59 & 11.40 \\
\hline DGR & 59.17 & 51.17 & 86.41 & 12.02 & 59.64 & 18.15 & 19.66 & 4 & 8.47 & 1 & 6.58 & 3.65 & 11.10 \\
\hline DND & 58.05 & 51.24 & 81.72 & 11.41 & 63.64 & 9.83 & 24.00 & 1 & 6.87 & 1 & 6.95 & 3.55 & 10.80 \\
\hline CKR1 & 72.65 & 62.85 & 144.05 & 14.79 & 62.25 & 11.24 & 27.42 & 4 & 7.80 & 2 & 5.38 & 3.65 & 13.20 \\
\hline CKR2 & 63.33 & 53.22 & 98.21 & 12.94 & 55.20 & 21.17 & 19.75 & 1 & 6.93 & 2 & 6.97 & 3.61 & 12.40 \\
\hline CKR3 & 59.48 & 54.05 & 82.71 & 10.21 & 61.85 & 10.26 & 25.50 & 1 & 8.07 & 1 & 7.67 & 3.65 & 13.40 \\
\hline CKR4 & 62.09 & 52.86 & 91.88 & 16.51 & 58.57 & 19.37 & 20.69 & 1 & 8.47 & 1 & 7.17 & 3.56 & 11.00 \\
\hline CKR5 & 59.53 & 50.42 & 84.80 & 11.48 & 57.29 & 17.06 & 20.86 & 1 & 8.67 & 2 & 8.03 & 3.55 & 11.30 \\
\hline BDM1 & 64.12 & 56.40 & 103.81 & 13.14 & 50.67 & 27.50 & 14.59 & 1 & 9.53 & 1 & 7.32 & 3.59 & 12.30 \\
\hline BDM2 & 68.01 & 62.89 & 126.44 & 16.31 & 50.37 & 28.00 & 15.33 & 1 & 8.67 & 2 & 7.41 & 3.57 & 13.80 \\
\hline BDM3 & 58.56 & 50.71 & 81.83 & 12.02 & 59.89 & 16.80 & 21.16 & 1 & 8.20 & 1 & 7.25 & 3.41 & 12.40 \\
\hline BDM4 & 61.35 & 50.00 & 87.12 & 12.66 & 53.50 & 27.11 & 15.82 & 1 & 8.67 & 1 & 7.07 & 3.48 & 12.40 \\
\hline BDM5 & 58.21 & 50.06 & 80.69 & 11.84 & 54.94 & 23.58 & 17.54 & 1 & 10.27 & 1 & 7.52 & 3.50 & 11.40 \\
\hline PNR1 & 62.69 & 52.89 & 96.39 & 13.21 & 55.90 & 19.05 & 19.69 & 1 & 7.73 & 1 & 7.63 & 3.53 & 12.20 \\
\hline PNR2 & 65.03 & 55.70 & 103.93 & 13.01 & 52.92 & 26.32 & 16.23 & 1 & 7.33 & 1 & 7.49 & 3.43 & 13.60 \\
\hline PNR3 & 63.60 & 55.56 & 103.04 & 13.39 & 56.03 & 23.38 & 18.32 & 1 & 8.67 & 1 & 7.07 & 3.46 & 13.20 \\
\hline DMR1 & 63.62 & 55.73 & 103.78 & 12.94 & 52.31 & 27.68 & 20.96 & 1 & 8.00 & 1 & 6.76 & 3.76 & 13.30 \\
\hline PNR5 & 63.47 & 54.60 & 99.96 & 13.83 & 40.90 & 35.47 & 17.95 & 1 & 7.73 & 1 & 7.42 & 3.42 & 11.40 \\
\hline DMR2 & 67.33 & 59.32 & 124.54 & 13.92 & 53.66 & 21.52 & 23.55 & 1 & 8.60 & 3 & 6.49 & 3.42 & 13.80 \\
\hline DMR3 & 63.45 & 56.90 & 104.16 & 15.47 & 65.63 & 14.18 & 30.16 & 1 & 8.27 & 1 & 7.16 & 3.05 & 13.10 \\
\hline DMR4 & 63.10 & 56.01 & 104.91 & 13.03 & 46.94 & 30.96 & 17.12 & 1 & 9.47 & 1 & 6.83 & 3.52 & 13.10 \\
\hline DMR5 & 60.65 & 53.41 & 95.71 & 11.86 & 54.06 & 21.63 & 22.13 & 4 & 8.07 & 1 & 6.24 & 3.88 & 11.80 \\
\hline CLL & 52.49 & 45.32 & 58.86 & 9.44 & 58.97 & 12.30 & 29.86 & 4 & 8.93 & 2 & 7.80 & 3.62 & 14.20 \\
\hline BRC & 58.85 & 50.47 & 82.65 & 13.14 & 50.72 & 28.06 & 18.88 & 1 & 7.87 & 1 & 6.55 & 3.57 & 13.90 \\
\hline ELG & 68.67 & 57.47 & 119.44 & 14.53 & 50.17 & 27.67 & 18.27 & 1 & 8.53 & 1 & 6.16 & 3.42 & 12.20 \\
\hline KVL1 & 66.14 & 56.47 & 110.46 & 14.36 & 53.73 & 18.25 & 25.96 & 2 & 6.73 & 1 & 6.65 & 3.35 & 13.60 \\
\hline KVL2 & 67.94 & 58.10 & 126.55 & 13.36 & 54.24 & 16.66 & 26.16 & 2 & 6.87 & 2 & 6.36 & 3.65 & 13.40 \\
\hline HCB1 & 60.32 & 53.73 & 92.63 & 12.92 & 51.90 & 25.35 & 20.11 & 1 & 6.73 & 1 & 6.90 & 3.47 & 10.30 \\
\hline HCB2 & 71.41 & 61.94 & 145.51 & 17.77 & 43.83 & 33.90 & 13.92 & 1 & 8.53 & 1 & 5.44 & 3.44 & 12.20 \\
\hline DKL & 65.77 & 62.93 & 125.60 & 15.00 & 45.31 & 30.67 & 16.58 & 1 & 6.07 & 1 & 6.74 & 3.66 & 14.00 \\
\hline YSL & 72.18 & 63.64 & 147.50 & 16.30 & 42.91 & 29.89 & 16.45 & 2 & 8.67 & 2 & 6.31 & 3.56 & 13.60 \\
\hline ULG & 72.07 & 66.40 & 154.44 & 16.56 & 52.50 & 21.04 & 22.15 & 2 & 6.40 & 2 & 5.83 & 3.73 & 12.80 \\
\hline GMS & 63.03 & 54.97 & 106.98 & 12.65 & 51.79 & 19.06 & 22.06 & 4 & 8.07 & 2 & 6.72 & 3.51 & 11.00 \\
\hline HMM & 63.36 & 57.24 & 108.67 & 12.19 & 55.26 & 11.45 & 27.58 & 4 & 8.80 & 2 & 8.09 & 3.47 & 13.80 \\
\hline ELM1 & 52.75 & 48.59 & 66.20 & 8.51 & 53.45 & 17.56 & 24.83 & 4 & 7.40 & 2 & 9.11 & 3.56 & 14.40 \\
\hline ELM2 & 66.94 & 59.96 & 121.23 & 14.33 & 48.71 & 25.21 & 19.74 & 1 & 6.47 & 2 & 7.82 & 3.53 & 12.30 \\
\hline $\mathrm{KCP}$ & 65.24 & 60.97 & 116.30 & 14.68 & 59.36 & 18.02 & 26.52 & 2 & 6.53 & 2 & 7.23 & 3.63 & 13.20 \\
\hline EYN & 64.80 & 57.19 & 108.34 & 12.10 & 53.39 & 26.24 & 21.18 & 2 & 9.67 & 2 & 7.78 & 3.57 & 13.10 \\
\hline ICM & 63.02 & 54.07 & 100.94 & 12.41 & 43.40 & 37.07 & 15.77 & 2 & 8.53 & 2 & 7.87 & 3.71 & 14.60 \\
\hline YLT & 62.97 & 54.26 & 100.55 & 13.51 & 56.43 & 12.81 & 24.89 & 2 & 8.60 & 3 & 7.29 & 3.63 & 12.40 \\
\hline
\end{tabular}

FHE: fruit height; FDI: fruit diameter; FWE: fruit weight; DSC: depth of stalk cavity; FSC-dL FSC-da FSC-db: fruit skin color; CFE: the color of flesh; NSE: number of seeds; ALO: aperture of locules; FFE: firmness of flesh; TSSC: total soluble solids content; CFE: white=1, cream=2, yellowish=3, greenish=4, pinkish=5, reddish=6; ALO: closed or slightly open=1, moderately open=2, fully open=3 


\subsection{Molecular results}

Fifteen different iPBS primers were used in the study (Table 4) to the determination of total of 60 samples for molecular analysis. Gel images of the iPBS 2392 which one of the primers used in the study, are given in Figure 1. As a result of molecular analyzes, 143 polymorphic bands were obtained.

Table 4- Description of used iPBS primers with names, sequence, annealing temperature, polymorphism rates and PIC values

\begin{tabular}{llccc}
\hline $\begin{array}{l}\text { Primer } \\
\text { names }\end{array}$ & $\begin{array}{l}\text { Primer } \\
\text { sequences }\end{array}$ & $\begin{array}{c}\text { Temperatures of } \\
\text { annealing }\left({ }^{\circ} \mathrm{C}\right)\end{array}$ & $\begin{array}{c}\text { Polymorphism } \\
\text { rate }(\%)\end{array}$ & $\begin{array}{c}\text { PIC } \\
\text { value }\end{array}$ \\
\hline 2075 & CTCATGATGCCA & $50^{\circ} \mathrm{C}$ & $94 \%$ & 0.34 \\
2381 & GTCCATCTTCCA & $50^{\circ} \mathrm{C}$ & $79 \%$ & 0.28 \\
2382 & TGTTGGCTTCCA & $50^{\circ} \mathrm{C}$ & $88 \%$ & 0.34 \\
2400 & CCCCTCCTTCTAGCGCCA & $50^{\circ} \mathrm{C}$ & $52 \%$ & 0.24 \\
2398 & GAACCCTTGCCGATACCA & $51^{\circ} \mathrm{C}$ & $71 \%$ & 0.29 \\
2252 & TCATGGCTCATGATACCA & $52^{\circ} \mathrm{C}$ & $71 \%$ & 0.39 \\
2277 & GGCGATGATACCA & $52^{\circ} \mathrm{C}$ & $55 \%$ & 0.23 \\
2375 & TCGCATCAACCA & $52^{\circ} \mathrm{C}$ & $60 \%$ & 0.16 \\
2392 & ATCTGTCAGCCA & $52^{\circ} \mathrm{C}$ & $88 \%$ & 0.36 \\
2085 & ATGCCGATACCA & $53^{\circ} \mathrm{C}$ & $47 \%$ & 0.21 \\
2095 & GCTCGGATACCA & $53^{\circ} \mathrm{C}$ & $15 \%$ & 0.11 \\
2232 & AGAGAGGCTCGGATACCA & $55^{\circ} \mathrm{C}$ & $17 \%$ & $17 \%$ \\
2237 & CCCCTACCTGGCGTGCCA & $55^{\circ} \mathrm{C}$ & $13 \%$ & 0.13 \\
2079 & AGGTGGGCGCCA & $60^{\circ} \mathrm{C}$ & $53^{\circ} \mathrm{C}$ & 0.10 \\
\hline
\end{tabular}

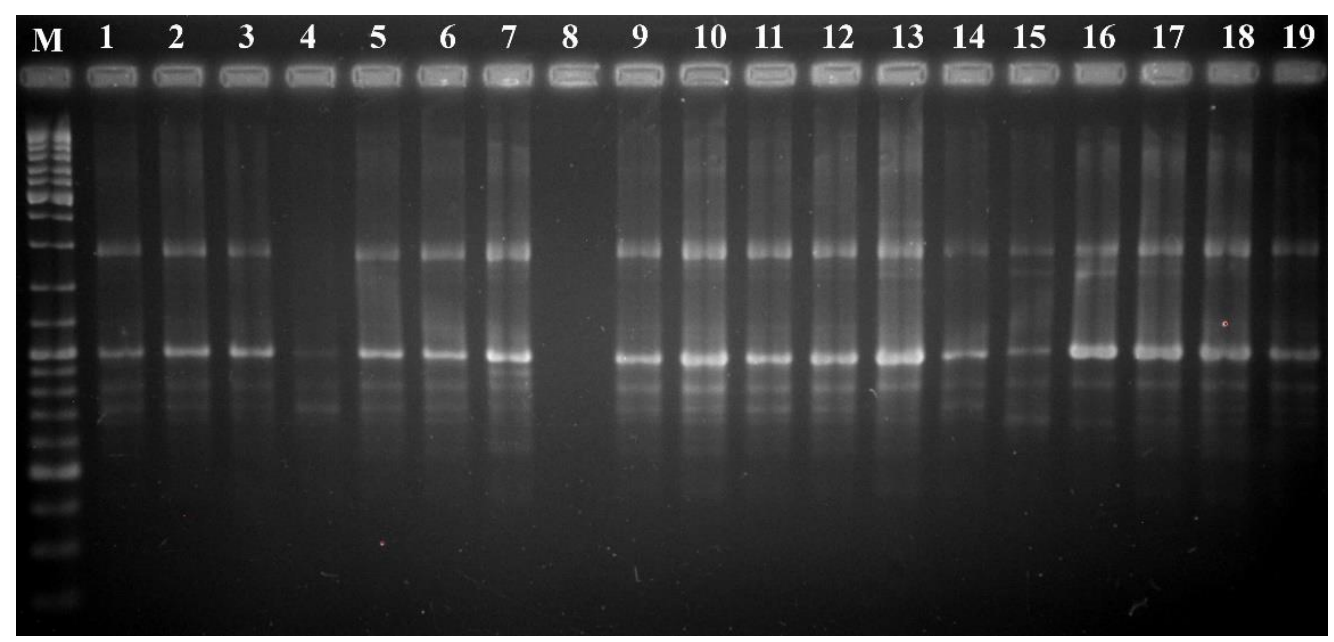

Figure 1- Gel image of iPBS 2392 primer, samples 1-19. 'M' stand for ladder (1 kb DNA ladder)

The main 48 samples were divided into 4 main branches and the similarity rates varied between 0.61-1.00 (Figure 2), with control groups these results extend to 7 main branches and the 0.54-1.00 similarity rates. Polymorphism rates of primers vary between 94\%-5\% and their PIC values vary between 0.10-0.34 (Table 4). The results of PCoA were similar to those of the cluster analysis. The first, second and third dimensions explained 23.6, 14.3 and $11.1 \%$ of the total variation making a total of $49 \%$ (Figure 3). 


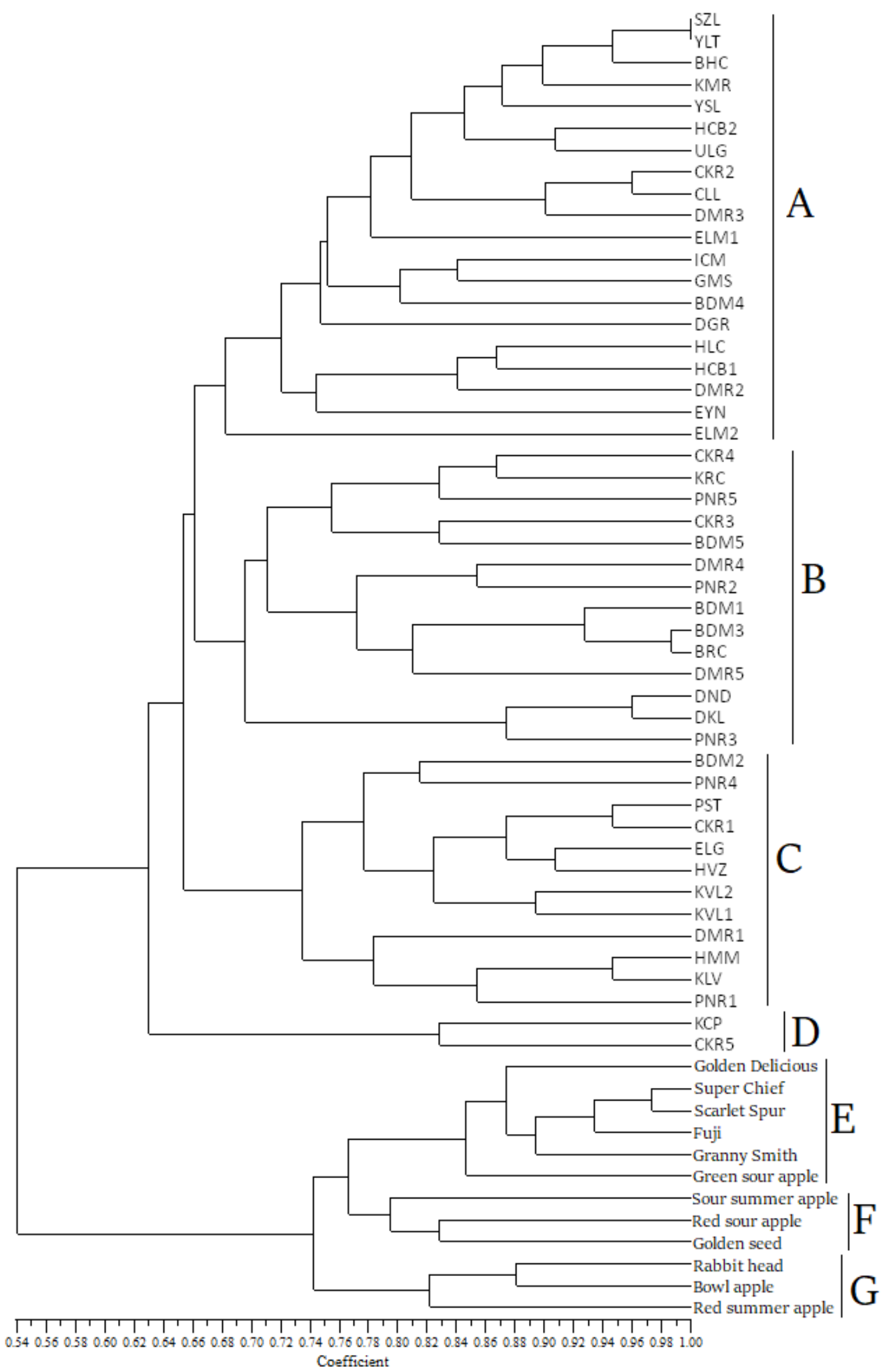

Figure 2- UPGMA dendrogram that shows the genetic diversity of samples with control groups using iPBS primers 


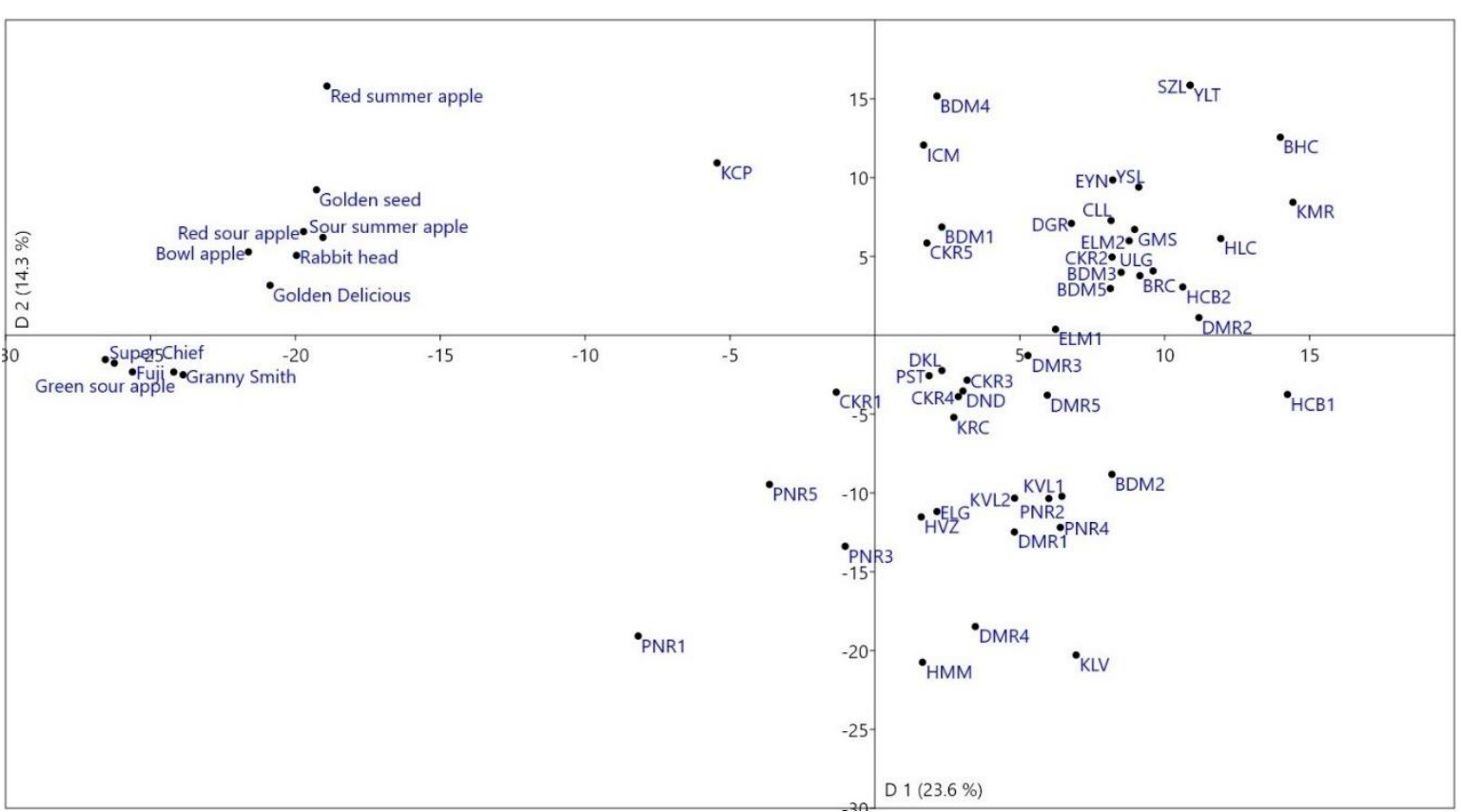

Figure 3- PCoA scatter plot showing the genetic diversity of a total 60 sample on first 2 dimension

\section{Discussion}

Eltez \& Kaska (1985) stated that fruit length 56.25-31.30 mm, fruit width 60.25-34.25 mm, fruit weight 180.20-55.45 gr, depth of stalk cavity 19.00-4.66 mm, total soluble solids content $16.65-11.20$, firmness of flesh of $10.05-6.48 \mathrm{~kg} / \mathrm{cm}^{2}$, the number of seeds 10.60 in their study with Amasya apples in Niğde. Although compared with the results of this study were shown prominent values among samples of the fruit diameter and fruit length, the overall values of the samples overlap with the value range. This is especially important for showing the consistency of the results, as the location and material of the two studies are similar. Under this information and with molecular results of our study, the diversity of area clarified. Coskun \& Askin (2016) stated that fruit length 64.70-53.90 mm, fruit width 76.60-64.90 mm, fruit weight 184.30-96.90 gr, total soluble solids content 14.20-11.30, firmness of flesh $8.73-6.43 \mathrm{~kg} / \mathrm{cm}^{2}$ in their study on local apple cultivars in Eğirdir. In this study, prominent samples were seen as fruit flesh hardness when pomological results compared with the previous study. Fruit flesh hardness important for crispness (De Belie et al. 2000). Although the two studies carried out different locations as materials both apples local to their location. Senyurt et al. (2015) stated that fruit weight of Amasya cultivar $96.43 \mathrm{~g}$, fruit length $54.95 \mathrm{~mm}$, fruit diameter $61.73 \mathrm{~mm}$, stalk pit depth $8.86 \mathrm{~mm}$, fruit flesh hardness $7.92 \mathrm{~kg} / \mathrm{cm}^{2}$, the weight of fruit 210.60-72.50 g, fruit width 79.70-57.40 mm, fruit length $71.15-50.68 \mathrm{~mm}$, fruit flesh hardness $10.00-6.80 \mathrm{~kg} / \mathrm{cm}^{2}$, total soluble solids content \% 14.20- 11.90, pH 4.24-3.08 values in their study in Egirdir different Amasya types. Compared to the result with earlier studies, although the samples in the total soluble solids content are prominent, the values overlapped.

The fruit size and weight are important for higher yield in production and also a wanted feature for most of the consumers. The color of the fruit skin is from the consumer's initial assessment and appeal of the product. Fruit flesh firmness is important to less damage during storage and transportation and also important for the crunchiness which is a trait consumer seeks when eating apples. Total soluble solids content was used in the selection and comparisons were made based on pomological results in this study because of its unique taste, sensation, and saturation (Arikan et al. 2015). Since the color of the fruit skin increases the attractiveness of the product for the consumer and high total soluble solids content value increases the taste of fruit and saturation of the fruit, it is important that the situation of samples used in the study show superiority (Chagne et al. 2014). Pomological results show that CKR2, DMR3, CLL, HCB2, YSL, ULG, ELM1, ICM samples stand out among other samples according to fruit size, fruit weight, fruit shell color, fruit flesh hardness, total soluble solids content.

Günes \& Durgac (2018) stated that the similarity rate between 0.39-0.72 as a result of the analysis using RAPD markers on local apples in the Gülnar region. Kaya et al. (2015) stated that the similarity ratio between $0.38-0.79$ as a result of the analysis using RAPD markers on local apple sources in Lake Van Basin. Masum et al. (2014) stated that the highest similarity between Marmara and Black Sea Region samples was 92.4\%, the lowest similarity between Black Sea-Central Anatolia region samples was $70.5 \%$ in the study conducted with local apples belonging to Marmara, Black Sea, Aegean, and Central Anatolian regions at Atatürk Central Horticultural Research Institute.

In this study, phenotypic results indicated diversity among collected genotypes as well as their distinction to the control group. But some of these results may be influenced environmentally due to genotypes collected from 29 different rural areas. Under these circumstances, the most coherent way to validation of phenotypic results is the utilization of molecular techniques 
to achieve more correct information about samples. In this study, iPBS is the chosen technique to manage this goal. The similarity ratio of this study 0.61-1.00 was compared with the previous studies and most of the samples shown higher similarities probably caused by location's effects on diversity but the results of molecular analysis have shown a variation among collected genotypes as well as their distinction to the control group. Although environmental influences can't be denied, both phenotypic and molecular results consistent with each other. Likewise, each sample that distinguishes the other can be used as a genetic source.

The study was conducted in an area where the total coverage approximately $1853 \mathrm{~km}^{2}$. Locations of BDM, PNR, DMR and CKR are relatively close to each other and contain 10 of 14 genotypes that under braches ' $\mathrm{B}$ '. On the other hand, this kind of phenomenon couldn't be seen for other main branches. Especially branches 'A' spread the whole area as well as 'B' on the UPGMA dendrogram. Branches ' $\mathrm{D}$ ' only contain two genotypes and the distance between these two genotypes $26.6 \mathrm{~km}$. In account of the most distance between two genotypes $59.72 \mathrm{~km}$ (HBC1 and HVZ), $26.6 \mathrm{~km}$ nearly the half of the distance between most distance genotypes. The location where the genotypes collected may affect to structure of braches on UPGMA dendrogram in some cases but the only reason underlying diversity among genotypes (Figure 2).

\section{Conclusions}

Morphological, pomological, and molecular differences were due to both location and genetics sources as the trees were found at varying heights in the elevation range of 1125-1726 m and sampled from the different parts of the province. Currently, limited studies have been carried out on apple with iPBS markers. Some studies were conducted on apple cultivars and mutants as well as other fruit species but this study is the first utilization of the iPBS markers system on apple genotypes. This research also supplied valuable information about this field via expanding the information about it. The current status can be improved by increasing and improving apple production in these rural areas with the use of modern agricultural techniques in production and storage stages, establish orchards with dwarf or semi-dwarf rootstocks and narrow plant spacing, perform agricultural practices on time with correct methods. According to information gathered from local peoples, these local apples in the province have decreased from the past and they are in danger of disappearing in the future. To preserve the plant material used in the study, 4 scions were taken from all of the trees and grafted on MM106 rootstocks and have been established a collection orchard in Niğde Ömer Halisdemir University Faculty of Agricultural Science and Technologies Faculty. Thus, in addition to the conservation of these resources, it will be possible to make controlled breeding for future studies.

\section{References}

Anonymous (2014). Researches on Determining the Current Situation of the Agri-Food Sector and Research-Development, Innovation Needs in Niğde and Surrounding Provinces. Ayhan Şahenk Faculty of Agricultural Sciences and Technologies (In Turkish)

Arıkan Ş, İpek M \& Pırlak L (2015). Determination of phenological and pomological characteristics of some apple varieties in Konya ecological conditions. Turkish Journal of Agriculture - Food Science and Technology 3(10): 811-815 DOI: 10.24925/turjaf.v3i10.811-815.402 (In Turkish)

Bakır M, Dumanoğlu H, Erdoğan V, Ernim C \& Macit T (2019). Characterization of wild apricot (Prunus armeniaca L.) genotypes selected from Cappadocia region (Nevșehir-Turkey) by SSR markers. Journal of Agricultural Sciences 25(4): 498-507 DOI: https://doi.org/10.15832/ankutbd.457850

Chagne D, Dayatilake D, Diack R, Oliver M, Ireland H, Watson A, Gardiner SE, Johnston J W, Schaffer J R \& Tustin S (2014). Genetic and environmental control of fruit maturation, dry matter and firmness in apple (Malus $\times$ domestica Borkh.). Horticulture Research 1(1): 1-12. DOI: $10.1038 /$ hortres.2014.46

Coskun S \& Askın M A (2016). Determination of Pomological and Biochemical Properties of Some Local Apple Varieties. SDU Faculty of Agriculture Journal 11(1): 120-131 (In Turkish)

De Belie N, De Smedt V \& De Baerdemaeker J (2000) Principal component analysis of chewing sounds to detect differences in apple crispness. Postharvest Biology and Technology 18(2): 109-119. DOI: 10.1016/S0925-5214(99)00070-8

Dellaporta S L, Wood J \& Hicks J B (1983). A plant DNA minipreparation: version II. Plant molecular biology reporter 1(4): 19-21 DOI: $10.1007 / \mathrm{BF} 02712670$

Demir Z \& Doğan I (2020). The Impact of Different Cover Crops, Mechanical Cultivation and Herbicide Treatment on The Soil Quality Variables and Yield in Apple (Malus domestica Borkh.) Orchard with a Coarse-Textured Soil. Journal of Agricultural Sciences 26(4): 452470 DOI: https://doi.org/10.15832/ankutbd.527445

Demirel U, Tindas I, Yavuz C, Baloch F S \& Caliskan M E (2018). Assessing genetic diversity of potato genotypes using inter-PBS retrotransposon marker system. Plant Genetic Resources 16(2): 137 DOI: 10.1017/S1479262117000041

Dunemann F, Kahnau R \& Schmidt H (1994). Genetic relationships in Malus evaluated by RAPD 'fingerprinting' of cultivars and wild species. Plant Breeding 113(2): 150-159 DOI: 10.1111/j.1439-0523.1994.tb00717.x

Eltez M \& Kaska N (1985). Selection of Highly Special Kaşel-Amasya Apple Types That Give Fruit Every Year in Niğde Region. Natural Science Journal 1(9) (In Turkish)

FAO (2020). FAOSTAT. Retrieved in May 2020 from http://www.fao.org/faostat/en/\#data/QC

Günes A \& Durgac C (2018). Morphological, Phenological, Pomological and Molecular Identification of Apple Genotypes Grown in Gülnar Region. Journal of Moleculer Biology and Biotechnology 2(1): 28-33 (In Turkish)

Hammer Ø, Harper D A \& Ryan P D (2001). PAST: Paleontological statistics software package for education and data analysis. Palaeontologia electronica 4(1): 9

Hancock J (2012). Plant evolution and the origin of crop species $3^{\text {rd }}$ Edition. CABI

Hinze L L, Fang D D, Gore M A, Scheffler B E, Yu J Z, Frelichowski J \& Percy R G (2015). Molecular characterization of the Gossypium diversity reference set of the US National Cotton Germplasm Collection. Theoretical and Applied Genetics 128: 313-327 DOI: $10.1007 / \mathrm{s} 00122-014-2431-7$ 
Hokanson S C, Szewc-McFadden A K, Lamboy W F \& McFerson J R (1998). Microsatellite (SSR) markers reveal genetic identities, genetic diversity and relationships in a Malus $\times$ domestica Borkh. core subset collection. Theoretical and Applied Genetics 97(5-6): 671-683 DOI: $10.1007 / \mathrm{s} 001220050943$

Hokanson S C, Lamboy W F, Szewc-McFadden A K \& McFerson J R (2001). Microsatellite (SSR) variation in a collection of Malus (apple) species and hybrids. Euphytica 118(3): 281-294 DOI: 10.1023/A:1017591202215

Kalendar R, Antonius K, Smýkal P \& Schulman A H (2010). iPBS: a universal method for DNA fingerprinting and retrotransposon isolation. Theoretical and Applied Genetics 121(8): 1419-1430 DOI: 10.1007/s00122-010-1398-2

Kaya T, Balta F \& Sensoy S (2015). Fruit quality parameters and molecular analysis of apple germplasm resources from van lake basin, Turkey. Turkish Journal of Agriculture and Forestry 39(6): 864-875 DOI: 10.3906/tar-1406-24

Kenis K \& Keulemans J (2005). Genetic linkage maps of two apple cultivars (Malus $\times$ domestica Borkh.) based on AFLP and microsatellite markers. Molecular Breeding 15(2): 205-219 DOI: 10.1007/s11032-004-5592-2

Kuras A, Antonius K, Kalendar R, Kruczy'nska D \& Korbin M (2013). Application of five DNA marker techniques to distinguish between five apple (Malus $\times$ domestica Borkh.) cultivars and their sports. The Journal of Horticultural Science and Biotechnology 88(6): 790-794 DOI: $10.1080 / 14620316.2013 .11513040$

Masum B, Ergul A, Kazan K, Akcay M E, Yuksel C, Bakır M, Mutaf F, Akpınar A E, Yaşasın A S \& Ayanoğlu H (2014). Genetic analysis of Anatolian apples (Malus sp.) by simple sequence repeat. Journal of Systematics and Evolution 52(5): 580-588 DOI: 10.1111/jse.12099

Milovanov A, Zvyagin A, Daniyarov A, Kalendar R \& Troshin L (2019). Genetic analysis of the grapevine genotypes of the Russian Vitis ampelographic collection using iPBS markers. Genetica 147(1): 91-101 DOI: 10.1007/s10709-019-00055-5

Ozbek S (1978). Special Fruit (Deciduous Fruit Species In Winter). Çukurova University Publication of Agriculture Faculty (In Turkish)

Rohlf F J (1998). NTSYS-pc, numerical taxonomy and multivariate system Version 2.0. Exet. Software. New York

SAS Institute Inc (2005). SAS User Guide; Sas/Stat, Version 8. SAS Inst. Inc.

Senyurt M, Kalkısım O \& Karadeniz T (2015). Pomological characteristics of some standard and local apple (Malus communis L.) varieties grown in Gümüşhane region. Academic Agriculture Journal 4(2): 59-64 (In Turkish)

Seymen T \& Polat M (2015). Determination of Phenological, Pomological Properties and Morphological Characterization of Some Amasya Apple Types (In Apple). Harran Journal of Agricultural and Food Science 19(3): 122-129. DOI: 10.29050/harranziraat.647181

Smolik M \& Krzysztoszek O (2010). Evaluation of genetic variability in choosen apple (Malus× domestica Borkh.) cultivars by ISSR-PCR analysis. Russian Journal of Genetics 46(7): 819-827 DOI: 10.1134/S1022795410070069

TUIK (2020). Retrieved in May 2020 from https://biruni.tuik.gov.tr/medas/?kn=92\&locale=tr

Tijskens L M M, Herold B, Zude M \& Geyer M (2007). How to choose criteria to harvest apples. The dynamics of maturity. In International Conference on Ripening Regulation and Postharvest Fruit Quality (796): 197-200. 10.17660/ActaHortic.2008.796.25

UPOV (2005). Apple, Guidelines For The Conduct Of Tests For Distinctness, Uniformity And Stability. TG/14/9

Zhang C, Chen X, He T, Liu X, Feng T \& Yuan Z (2007). Genetic structure of Malus sieversii population m Xinjiang, China, revealed by SSR markers. Journal of Genetics and Genomics 34(10): 947-955 DOI: 10.1016/S1673-8527(07)60106-4

Zhou Z Q \& Li Y N (2000). The RAPD evidence for the phylogenetic relationship of the closely related species of cultivated apple. Genetic Resources and Crop Evolution 47(4): 353-357 DOI: 10.1023/A:1008740819941

(C) 2022 by the author(s). Published by Ankara University, Faculty of Agriculture, Ankara, Turkey. This is an Open Access article distributed under the terms and conditions of the Creative Commons Attribution (CC BY) license (http://creativecommons.org/licenses/by/4.0/), which permits unrestricted use, distribution, and reproduction in any medium, provided the original work is properly cited. 